\title{
Commissioning and first results of the Fermilab Muon Campus
}

\author{
Diktys Stratakis, Brian Drendel, and James P. Morgan \\ Fermi National Accelerator Laboratory, Batavia, Illinois 60510, USA \\ Michael J. Syphers and Nathan S. Froemming \\ Northern Illinois University, DeKalb, Illinois 60115, USA
}

(Received 1 October 2018; published 3 January 2019)

\begin{abstract}
In the following years, the Fermilab Muon Campus will deliver highly polarized muon beams to the Muon $g-2$ experiment. The Muon Campus contains a target section wherein secondaries are produced, the delivery ring which separates the muons from the rest of the beam, and a sequence of beam lines that transports them to the Muon $g-2$ storage ring. Here, we report the first results of beam measurements at the Muon Campus with an emphasis on the key achievements that have contributed to the successful beam delivery to the Muon $g-2$ experiment. These achievements include the production of an intense secondary beam from the target, its transport over $2 \mathrm{~km}$, the successful monitoring of muons from the available diagnostics, and the development of techniques for measuring the transverse optics. We also present detailed comparisons between the experimental data and simulation and discuss the similarities and differences observed.
\end{abstract}

DOI: 10.1103/PhysRevAccelBeams.22.011001

\section{INTRODUCTION}

The Muon $g-2$ experiment at Fermilab [1] will measure the anomalous magnetic moment of the muon, $\alpha=(g-2) / 2$, with an unprecedented precision of 0.14 parts per million. Here, $g$ is the gyromagnetic moment of the muon; for a pure Dirac particle, $g=2$, and hence $\alpha$ refers to the anomalous relative deviation from this value. To perform the experiment, a polarized beam of positive muons is injected into a storage ring with a vertical magnetic field [2]. Since the positron direction from the weak muon decay is correlated with the spin of the muon, the precession frequency is measured by counting the rate of positrons above an energy threshold versus time at detectors located about the ring circumference. The $g-2$ value is then proportional to the precession frequency divided by the magnetic field of the storage ring [3].

The Muon $g-2$ experiment runs at the new Fermilab Muon Campus [4]. A number of simulation studies [5-7] have shown that the Muon Campus has the potential to deliver highly polarized muons up to 21 times the statistics of the equivalent Brookhaven experiment [8]. For the Fermilab Muon Campus operations, protons accelerated in the upgraded linac and booster synchrotron [9] are adiabatically rebunched [10] in the recycler synchrotron

Published by the American Physical Society under the terms of the Creative Commons Attribution 4.0 International license. Further distribution of this work must maintain attribution to the author(s) and the published article's title, journal citation, and DOI. and directed to an Inconel target [11]. Secondary beam pions then travel down a $280 \mathrm{~m}$ transfer line and then around the delivery ring [12], during which the pions decay into muons. The final muon beam is ejected and sent on to the storage ring of the Muon $g-2$ experiment [13]. The transport through the delivery ring (DR) is advantageous, as it increases the gap between the "heavy" protons and "light" muons, allowing the protons to be removed from the beam.

In this paper, we report the first experimental results of the Fermilab Muon Campus with an emphasis on the milestones that have demonstrated the Muon Campus capability of delivering a beam to the Muon $g-2$ experiment. These milestones include the interaction of the primary proton beam with the target, the generation and transport of muons over long sections, the monitoring of secondaries from the available diagnostics, and the development of techniques for measuring the beam optics. We also compare beam measurements with predictions from simulations and show that our model can describe the physics of the beam delivery system within a reasonable level of agreement.

The outline of the paper is as follows. In Sec. II, we will overview the Muon Campus beam lines and the available instrumentation as well as the computational tools developed in order to study its performance. In Sec. III, we will overview the commissioning steps of the Fermilab Muon Campus. Then, in Sec. IV, we will present our experimental results and compare them with predictions from numerical simulations. In the last part of the paper (Sec. V), we will detail our conclusions. 


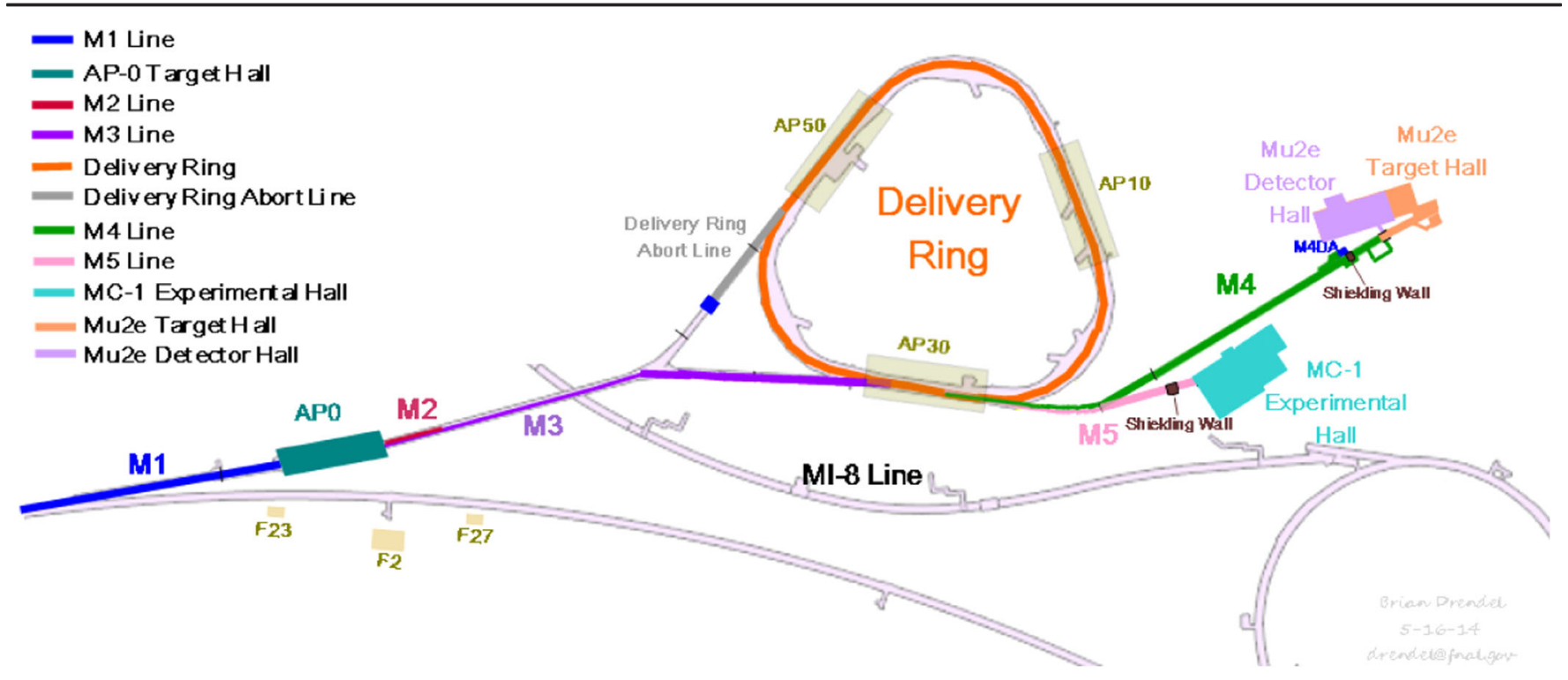

FIG. 1. A schematic representation of the Muon Campus accelerator complex that is used by the Muon $g-2$ experiment. Secondaries are produced on a target that then travel through the M2 and M3 lines, which are designed to capture as many $3.1 \mathrm{GeV} / c$ muons from pion decays as possible. The beam is injected into the DR, wherein a kicker is used to remove the protons, the resulting muon beam is then extracted into the M4 line, and the muon beam is eventually transferred to the M5 line that leads to the muon storage ring (enclosed in the MC1 hall). The combined M2 and M3 line and M4 and M5 line lengths are 280 and 130 m, respectively, along with the DR that has a circumference of $505 \mathrm{~m}$.

\section{LATTICE DESIGN, BEAM CONTROL, AND MODELING}

Figure 1 shows a schematic layout of the Fermilab Muon Campus. While a more detailed description of all beam lines can be found elsewhere [14], we will review here their main features. Protons with a kinetic energy of $8 \mathrm{GeV}$ are delivered through the M1 beam line to an Inconel target [15] at AP0 and produce a beam of secondary particles that is most significantly composed of protons, pions, positrons, and deuterons. During the Muon $g-2$ experiment, 16 pulses are sent to the target with a frequency of $12 \mathrm{~Hz}$. More details about the parameters of the primary input beam can be found in Table I. The positive secondaries are focused by a lithium lens and then momentum-selected via a downstream pulsed dipole magnet (PMAG). The PMAG selects $3.1 \mathrm{GeV} / c$ positive particles and bends them $3^{\circ}$ into the M2-line channel. The M2 line is $50 \mathrm{~m}$ long and consists of a sequence of FODO cells with a $120^{\circ}$ phase advance. Further downstream, a second dipole magnet provides another $3^{\circ}$ bend to align the beam with the M3-line

TABLE I. Primary beam parameters at the production target.

\begin{tabular}{lc}
\hline \hline Parameter & Value \\
\hline Intensity per pulse & $10^{12}$ \\
Number of pulses per cycle & 16 \\
Cycle length (s) & 1.4 \\
Frequency $(\mathrm{Hz})$ & 12 \\
Kinetic energy $(\mathrm{GeV})$ & 8.0 \\
Beam size at the target $(\mathrm{mm} \times \mathrm{mm})$ & $0.22 \times 0.24$ \\
\hline \hline
\end{tabular}

trajectory. The M3 line continues with a series of FODO cells with a $90^{\circ}$ phase advance for $100 \mathrm{~m}$, wherein a horizontal right bend, provided by a specialized insertion created from two $9.25^{\circ}$ dipole bends, aligns the beam to the downstream leg of the M3 line. This line continues with another sequence of FODO cells at $72^{\circ}$ phase advance for nearly $130 \mathrm{~m}$, and, after another horizontal $5^{\circ}$ bend, the beam is finally injected vertically into the DR. A layout of this injection scheme is illustrated in Fig. 2. Vertical injection into the DR is achieved with a bend produced by a combination of a $\mathrm{C}$ magnet (CMAG), followed by a large-aperture focusing quad (Q303) and a pulsed magnetic septum dipole. Then, two kicker modules (IKIK1 and IKIK2) send the trajectory onto the DR closed orbit. It is important to emphasize that the injection section in Fig. 2 contains the narrowest apertures along all Muon Campus beam lines, and therefore any upstream mismatches can significantly harm the performance. After injection, the beam circulates the DR several turns to achieve a longitudinal separation between protons and muons, due to their velocity difference. After the fourth turn, a kicker magnet is used to remove the proton beam, and a separate kicker extracts the muon beam into the M4 line.

Injection from the M3 line and extraction to the M4 line takes place in the same straight section, with the latter happening in the downstream half. For extraction, the beam will be first kicked out of the DR closed orbit via two kicker magnets; then, with the aid of a Lambertson magnet and vertical bending magnets, the beam is extracted upward out of the DR. After $30 \mathrm{~m}$ in the M4 line, the beam bends upwards one more time into the M5 


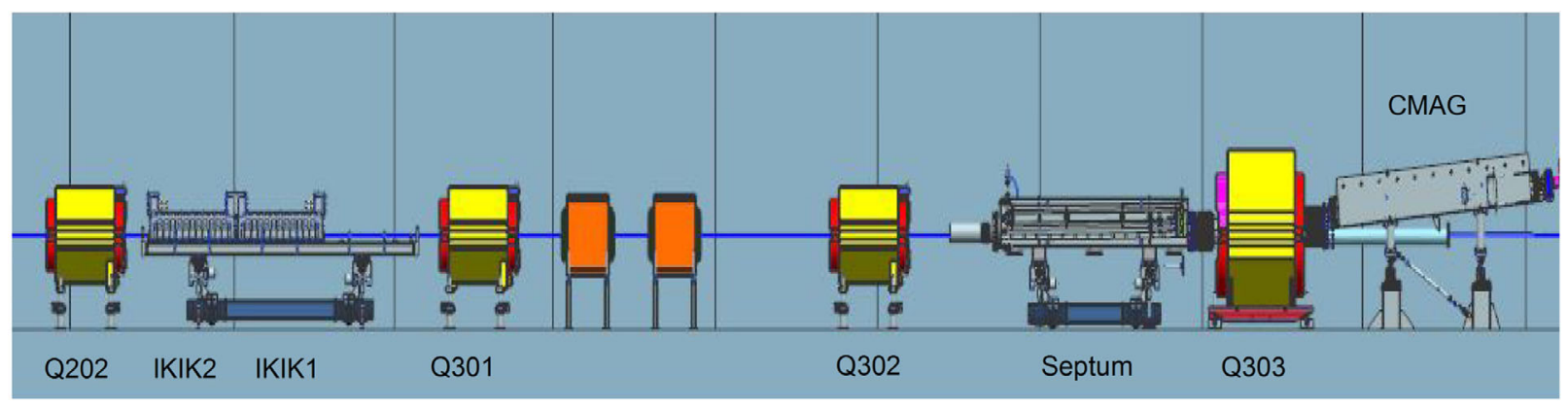

FIG. 2. Conceptual design for injection into the DR, where the beam direction is from right to left. The yellow elements are quads. The two orange objects between quads 302 and 301 are steering dipoles. CMAG is a vertical bending magnet and is the last element of the M3 line.

line and then levels off and continues toward the storage ring of the Muon $g-2$ experiment. The M5 line is nearly $100 \mathrm{~m}$ long and contains a $27.1^{\circ}$ horizontal bend string that provides the proper entry position and angle into the storage ring. Roughly $10 \mathrm{~m}$ before the end of the M5 line, there is a strong-focusing and tunable final focus section, consisting of four quadrupole magnets, that provides optical matching to the storage ring.

Several devices are strategically placed along the Muon Campus in order to properly diagnose the beam properties [16]. The primary proton beam (upstream of the target) is monitored with toroids, beam position monitors, and beam loss monitors. Since the secondary beam has an intensity that is at least 2 orders of magnitude less than that of the primary, such devices are not viable for the secondary beam, and therefore its diagnosis fully relies on ion chambers (ICs), secondary emission monitors (SEMs), and proportional wire chambers (PWCs). Table II shows the location of the ICs, SEMs, and PWCs along the Muon Campus starting from the target and extending all the way to the M5 line. Note that, since the main interest of this study is the secondary beam, we will give more emphasis on IC, SEM, and PWC monitors.

SEMs are mainly used to measure beam profiles in the first part of the M2 and M3 lines, wherein a total of eight SEMs are strategically placed at various positions. The SEMs have a set of signal titanium foils that are aligned in both horizontal and vertical planes. In each plane, there are 30 foils with a $2 \mathrm{~mm}$ width and a $3 \mathrm{~mm}$ spacing. The SEMs collect the charge from each of the foils and convert it to a voltage value proportional to the total charge collected. The data are transferred to the user for analysis and display. Toward the end of the M3 line, the beam profiles are measured using PWCs. PWCs are more sensitive than SEMs, since they have the capability to measure beam intensities down to the $10^{3}$ range per $12 \mathrm{~Hz}$ pulse. PWCs contain a group of signal wires that are aligned in both horizontal and vertical planes. Each group contains 48 gold-plated tungsten wires with a $10 \mu \mathrm{m}$ diameter and a $2 \mathrm{~mm}$ spacing. Similar to the SEMs, the charge from each wire is collected and converted to a voltage value proportional to the charge sampled. Finally, the intensity of the secondary beam will be measured with ICs. ICs can measure beam intensities with an accuracy of $\pm 5 \%$ with as few as $10^{5}$ particles per $12 \mathrm{~Hz}$ pulse. They are implemented

TABLE II. Location of available beam diagnostics along the Muon Campus. For the DR diagnostics, only their location during the first pass is shown. During operations for the Muon $g-2$ experiment, the beam is circulating the DR four times. Note that $\mathrm{S}=0$ is at the downstream edge of the target.

\begin{tabular}{|c|c|c|c|}
\hline Beam line & $\mathrm{S}(\mathrm{m})$ & Type & Detector ID \\
\hline M1 & -0.43 & SEM & TARGET-SEM \\
\hline M2 & 20.45 & IC & 804 \\
\hline $\mathrm{M} 2$ & 21.02 & SEM & 804 \\
\hline $\mathrm{M} 2$ & 37.98 & SEM & 810 \\
\hline M3 & 51.76 & SEM & 706 \\
\hline M3 & 77.26 & SEM & 711 \\
\hline M3 & 121.30 & SEM & 719 \\
\hline M3 & 163.41 & SEM & 726 \\
\hline M3 & 173.24 & SEM & 729 \\
\hline M3 & 234.83 & SEM & 740 \\
\hline M3 & 235.21 & $\mathrm{IC}$ & 740 \\
\hline M3 & 259.55 & PWC & 744 \\
\hline M3 & 269.76 & PWC & 748 \\
\hline DR & 289.78 & PWC & 301 \\
\hline DR & 306.90 & PWC & 204 \\
\hline DR & 329.01 & $\mathrm{IC}$ & 209 \\
\hline DR & 441.43 & IC & 105 \\
\hline DR & 441.70 & PWC & 105 \\
\hline DR & 487.62 & PWC & 607 \\
\hline DR & 607.84 & PWC & 505 \\
\hline DR & 640.03 & PWC & 403 \\
\hline M4 & 2332.31 & PWC & 900 \\
\hline M4 & 2340.74 & IC & 902 \\
\hline M4 & 2345.11 & PWC & 904 \\
\hline M5 & 2361.75 & PWC & 000 \\
\hline M5 & 2375.57 & PWC & 005 \\
\hline M5 & 2388.78 & PWC & 011 \\
\hline M5 & 2403.96 & PWC & 014 \\
\hline M5 & 2437.53 & PWC & 020 \\
\hline M5 & 2444.33 & PWC & 021 \\
\hline M5 & 2456.30 & PWC & 025 \\
\hline M5 & 2456.50 & IC & 025 \\
\hline
\end{tabular}


at the M2-M3 lines, the DR, and the M4-M5 lines. In order to mitigate scattering effects, SEMs, PWCs, and ICs are placed on a motorized drive that allows them to be moved in or out of the beam path whenever it is necessary during the experiment.

The interaction of the proton beam with the target was modeled using both MARS [17] and G4beamline [18], and a $6 \mathrm{D}$ distribution of secondary particles was obtained at the exit of the production target. G4beamline is an open-source particle simulation program that acts as a front end for the Geant4 [19] simulation package. The simulation includes key physical processes such as pion decay, muon decay, and precession of the muon spin. The performance of the Muon Campus beam lines downstream of the production target was simulated using G4beamline. In the simulation, virtual detectors where placed at the location of available diagnostics. As a result, it was possible to evaluate the population of secondaries and their $6 \mathrm{D}$ phase space distribution as well as the beam profiles at several locations and compare them with experimental data. While special care was taken to model the physical apertures as closely as possible, in some cases approximations have to be made, especially for the asymmetrical apertures of the injection quadrupoles, the kickers, and the septum magnet. Finally, routine beam optics studies such as the evolution of the Twiss parameters and dispersion along the lines was carried out using MADX [20]. Notice that all simulation performances reported hereafter correspond to the baseline design parameters, which are discussed in more detail in Ref. [14].

\section{MUON CAMPUS COMMISIONING}

Commissioning of the Fermilab Muon Campus begun in April 2017 and consisted of three phases. In the first phase, an $8 \mathrm{GeV}$ proton beam from the recycler bypassed the target, then entered the DR via the M3 line, and was extracted into the upstream M4 line. Note that the intensity of this beam was 2 orders of magnitude higher than the secondary beam. As a result, this part of the commissioning provided a good test bed for cross-checking the optics and available beam line instrumentation along the Muon Campus. For the second phase, the primary beam was sent to the target with the goal to commission the newborn $3.1 \mathrm{GeV} / c$ secondary beam. To save experimental time, the secondary beam did not travel around the DR but rather passed straight through the M4 and M5 lines and into the storage ring of the Muon $g-2$ experiment [21,22]. In the third and last phase of commissioning, the primary beam was sent to the target, the resulting $3.1 \mathrm{GeV} / c$ secondary beam was injected into the DR via the M3 line, protons were removed after the fourth turn, and finally the beam was sent in the storage ring via the M4 and M5 lines. This scenario is illustrated in Fig. 1 and will be the primary focus of this paper.

\section{EXPERIMENTAL AND NUMERICAL RESULTS}

\section{A. Production target}

Figure 3 displays the measured SEM profile of the incoming primary proton beam just upstream of the target. The histograms are raw data, while the red curves are Gaussian fits to the data. We can see that the beam retains a Gaussian profile with $\sigma_{x}=0.22 \mathrm{~mm}$ and $\sigma_{y}=0.24 \mathrm{~mm}$ in the horizontal and vertical planes, respectively. This spot size is slightly higher compared to $0.15 \mathrm{~mm}$ of the baseline design. To further examine the implications of this, in Fig. 4 we plot the simulated relative pion yield downstream from the lithium lens as a function of the primary beam spot size at the target. One can see that if the spot size is reduced from 0.55 to $0.15 \mathrm{~mm}$, a $15 \%$ increase in pion production can be achieved. As a result, the spot size is a key parameter that governs the final secondary yield in the Muon Campus.
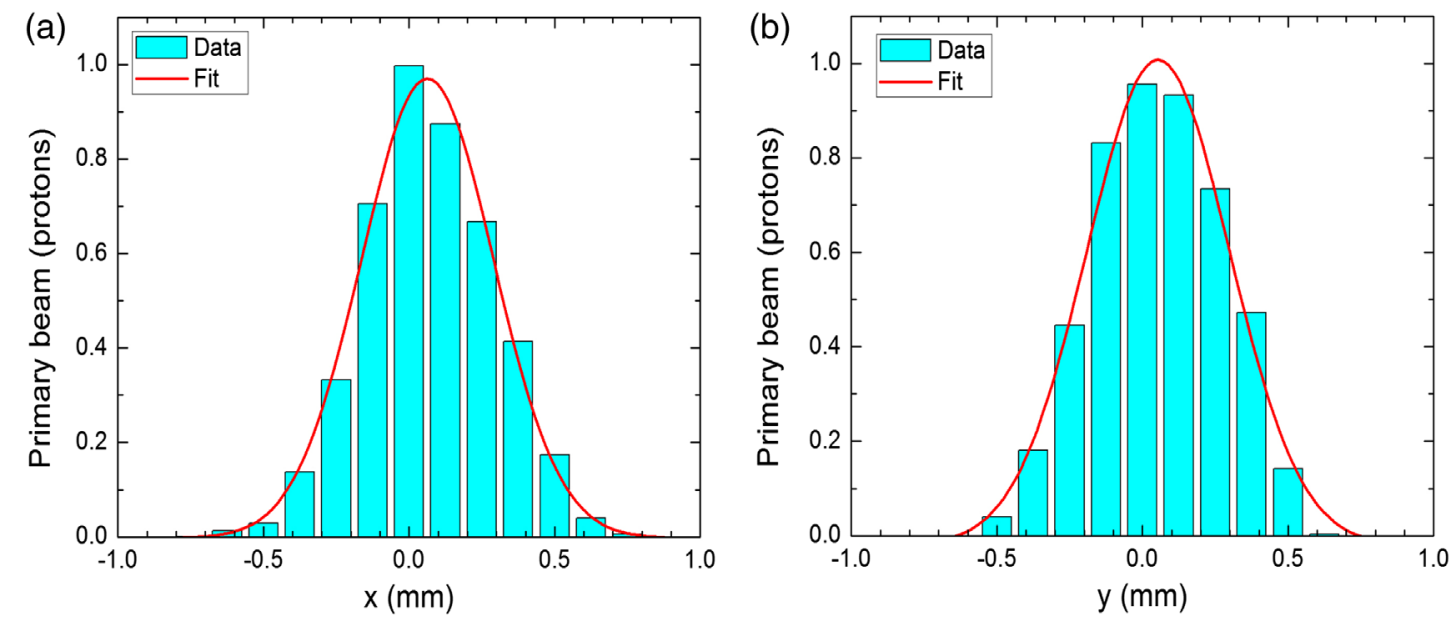

FIG. 3. Measured profile of the incoming primary proton beam just upstream of the production target (a) horizontal beam profile and (b) vertical beam profile. The histograms are raw data at the TARGET-SEM, while the red curve is a Gaussian fit. The fit to the data reveals that the beam spot size at the target is $\sigma_{x}=0.22 \mathrm{~mm}$ and $\sigma_{y}=0.24 \mathrm{~mm}$. 


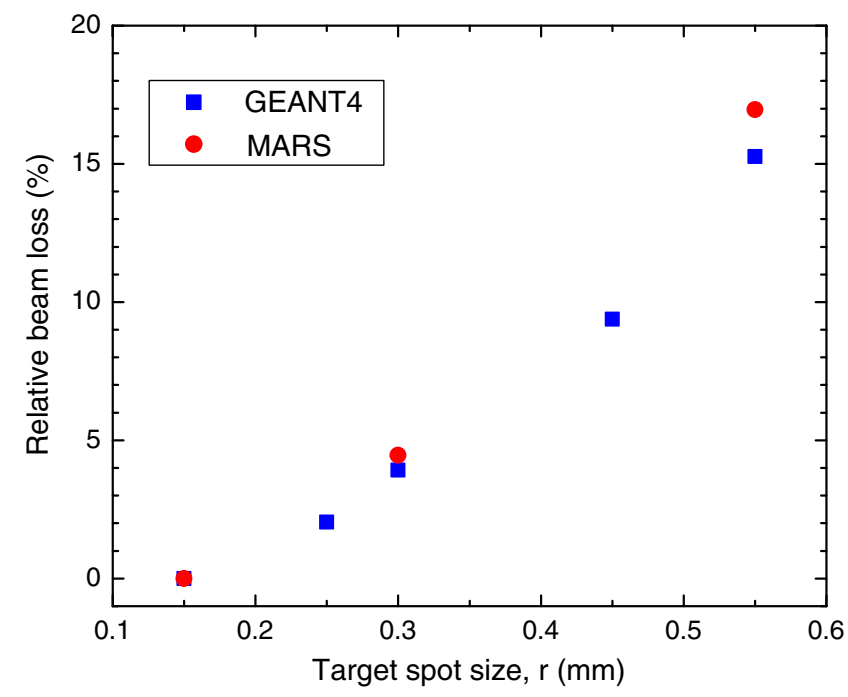

FIG. 4. Relative loss of the number of pions at the downstream face of the lithium lens as a function of the beam rms size at the target. Equal size in both horizontal and vertical planes is assumed. The software cut on pion momentum was limited to $2.5 \%$ around the central momentum of $3.1 \mathrm{GeV} / c$. Note that the baseline design considers a $0.15 \mathrm{~mm}$ beam spot size at the target.

In comparison to the baseline design, however, we anticipate that the spot size achieved in our experiment should result in no perceptible difference in the downstream secondary beam rates. Quantitatively, both MARS and G4beamline showed that a spot size of $0.20-0.25 \mathrm{~mm}$ resulted in a $<3 \%$ difference compared to the baseline design performance. Note that the source of a small discrepancy

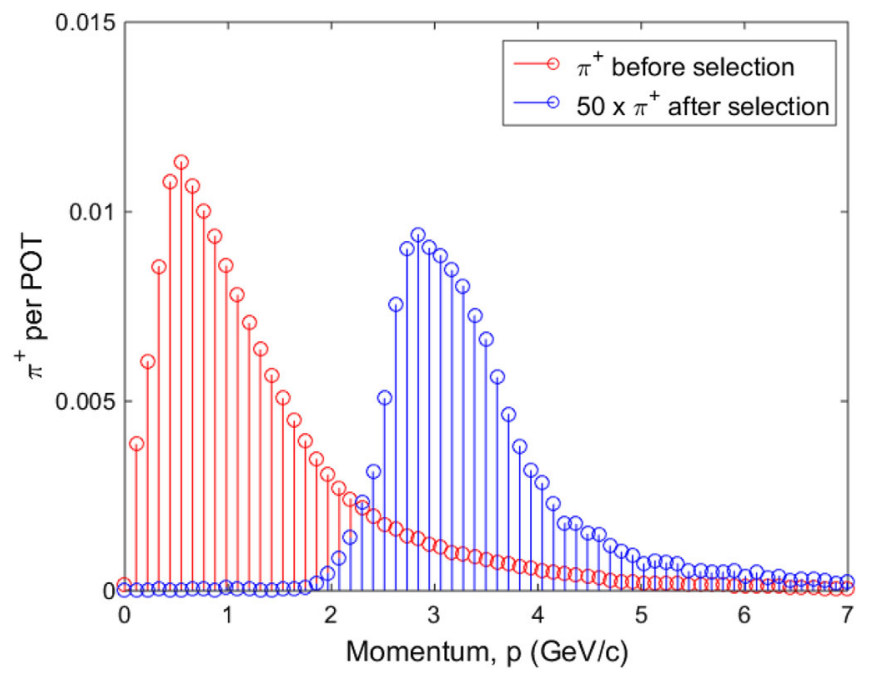

FIG. 5. Red: Momentum histogram of the pion beam a few millimeters after the target downstream edge. Blue: Momentum histogram downstream of the PMAG. The selection process is clearly shown. Note that the distribution shows a slight asymmetry, meaning that the largest population of secondary particles is concentrated at lower momenta. For visual purposes, the number of pions after the PMAG is multiplied by a factor of 50 . between MARS and GEANT4 results is traced back to the lithium lens which in MARS is modeled with more detail.

The beam-target interactions were further modeled, and a particle distribution was created $0.77 \mathrm{~m}$ upstream of the PMAG and propagated further downstream using G4beamline. Figure 5 displays the momentum distribution of pions before and after the PMAG, wherein the vertical axis is scaled to the protons on target (POT). Initially, the beam has a wide momentum spectrum which extends all the way to $7 \mathrm{GeV} / c$ (red). Conversely, the momentum distribution is closely centered to the desired $3.1 \mathrm{GeV} / c$ momentum after the beam passes the PMAG (blue). This indicates the central role of PMAG in shaping the momentum distribution. Notice further that the momentum distribution in blue exhibits a slight asymmetry. This fact is not surprising, since the vast majority of the pions entering the dipole magnet have a momentum that is less than the desired $3.1 \mathrm{GeV} / c$, and the implication of this will be discussed in more detail in the next section.

\section{B. Muon capture and transport (M2 and M3 beam lines)}

The purpose of the M2 and M3 lines is to capture muons with momentum $3.1 \mathrm{GeV} / c$ from pion decays and then direct them towards the DR. A key feature of the M2 and M3 lines is that they have a high quadrupole density so as to maximize the capture efficiency of the secondary beam. As a result, the beta functions vary smoothly and do not exceed $20 \mathrm{~m}$ in both vertical and horizontal planes for most part of the beam line. In addition, the relative narrow momentum acceptance of the lattice, i.e., $\Delta p / p \approx \pm 4 \%$, ensures that most muons are born from forward-decayed pions, generating a muon beam with high longitudinal polarization. Quantitatively, 71\% of the pions are expected to decay, therefore making this part of the channel the location wherein most muons are born. The simulated beam intensity as a function of the distance along the M2 and M3 lines is shown in Fig. 6. One can see that the main species leaving the target are protons, pions, muons, and positrons. Notice that there is a small fraction of deuterons traveling as well, but their rates are considerably smaller compared to positrons and therefore are not displayed in the figure.

A careful examination of Fig. 6 reveals that there is a substantial drop in intensity as the beam travels downstream of the target. This fact is not surprising, since only particles near $3.1 \mathrm{GeV} / c$ are selected, and, as the beam travels further downstream, pions decay into muons, where only the daughter muon near $3.1 \mathrm{GeV} / c$ is getting accepted. The additional drop near $160 \mathrm{~m}$ is from momentum collimation at the two $9.25^{\circ}$ horizontal dipole magnets that align the beam trajectory toward the DR [14]. The black square corresponds to the measured intensity at IC 740 at the end of the line. One can see that the measured value agrees well with the predicted intensity from the 


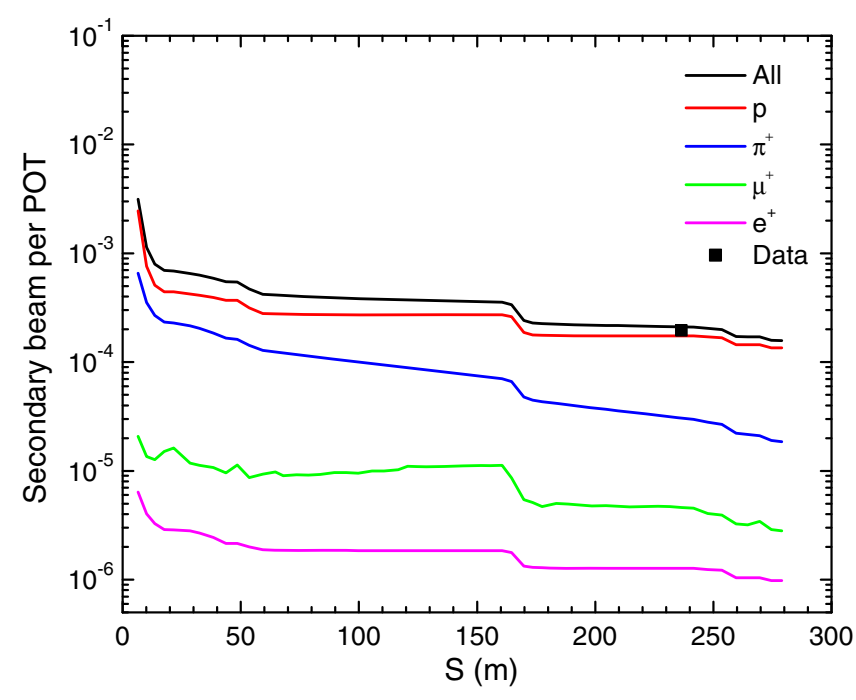

FIG. 6. Simulated performance of secondaries along the Muon Campus M2 and M3 lines for the baseline design specifications. The overall number of secondaries is scaled to the POT, and only particles within $-52 \mathrm{~mm}<x$ or $y<52 \mathrm{~mm}$ are recorded. The black square shows the measured beam intensity at IC 740 . Notice that the lattice is designed to have a narrow momentum acceptance of $\Delta p / p= \pm 4 \%$.
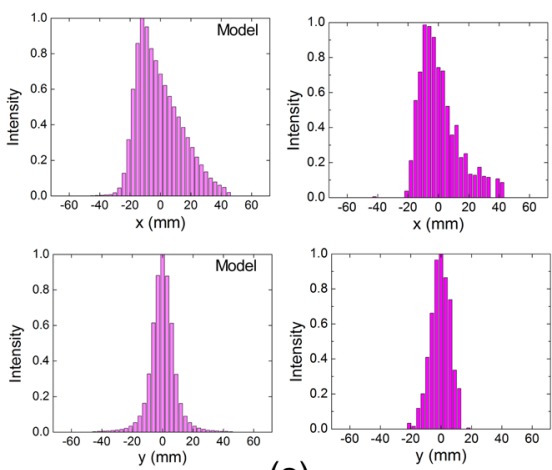

(a)
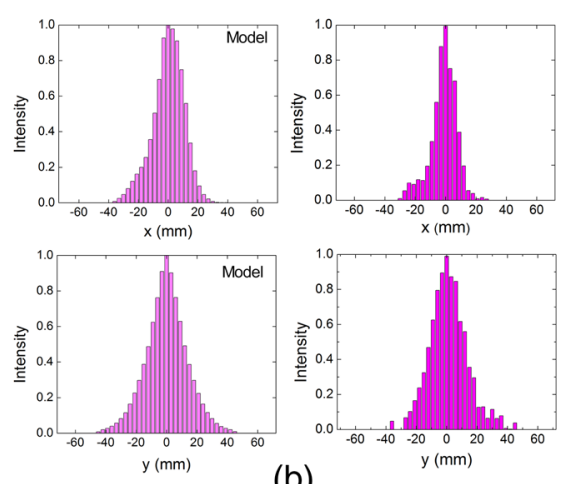

(b) simulation. The significance of this result is that it demonstrates that the target and the following beam lines can produce, capture, and deliver to the DR a secondary beam within the baseline design specifications.

The transverse beam profiles at different locations along the M2 and M3 lines are shown in Fig. 7. For all subfigures, the top and second rows show the horizontal and vertical profiles, respectively. Furthermore, the first column displays the simulated profile, while the second column displays the measured one. Unlike the vertical profiles, the horizontal beam profiles deviate from the conventional Gaussian shape especially at the first half of the line. This is a direct consequence of the asymmetric nature of the incoming secondary beam from the target (see Fig. 5). As a result, particles with different momentum will follow different paths in phase space as they propagate through areas with dispersion. Since the M2 and M3 lines contain mostly horizontal bends, the introduced dispersion causes the deviations from the Gaussian shape to be mostly evident in the horizontal plane. Interestingly, a similar trend is also predicted by the model. Overall, one can see from Fig. 7 that the agreement between the experiment and the simulation is reasonable; however, it was less pronounced towards the end of the M3. The source of this
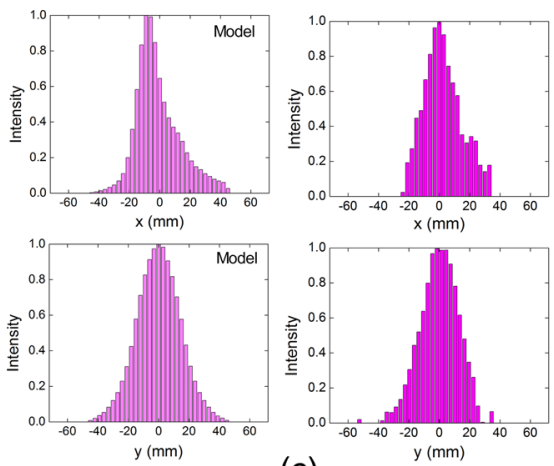

(c)
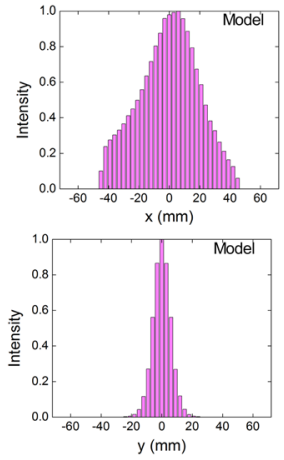
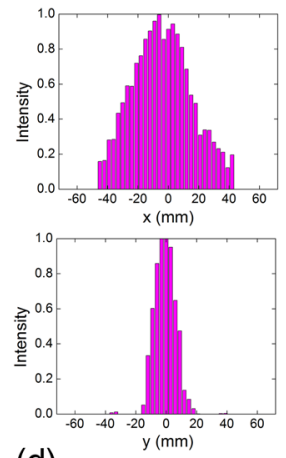

(d)
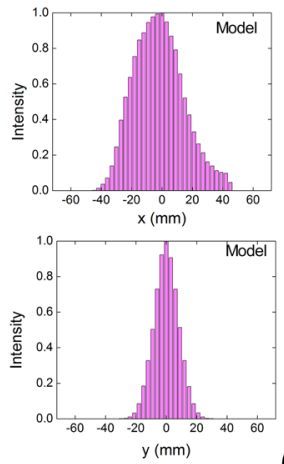

(e)
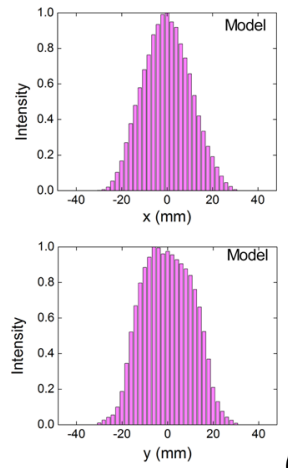

(f)
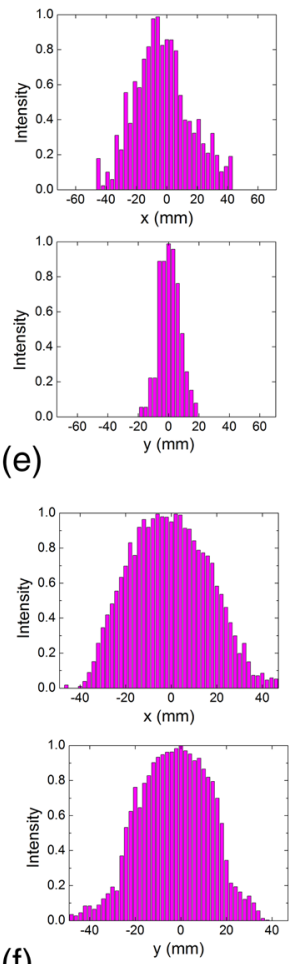

FIG. 7. Transverse beam profiles at different instrumentation locations along the M2 and M3 lines. (a) At SEM804. (b) At SEM810. (c) At SEM706. (d) At SEM711. (e) At SEM726. (f) At PWC744. Note that, in all subfigures, the top and second rows show the horizontal and vertical profiles, respectively. Furthermore, the first column shows the simulated profile, while the second column shows the measured one. 
discrepancy has been traced back to quadrupole steering errors, which, although in the submillimeter range, can perturb the beam distribution, resulting in a divergence from the design optics that is accompanied by a dispersion wave that can persist over look sections [23].

Next, using G4beamline we examine the behavior of individual secondary particles. We choose the same locations as in Fig. 7, and the corresponding beam profiles are shown in Fig. 8. A common behavior between protons and pions is observed, since their profiles exhibit similar asymmetry in the first half of the line, where the proton population dominates. Conversely, the muon profiles resemble a shape that is closer to a Gaussian. This is a direct consequence of the fact that most muons are produced from pion decay and therefore have a very wide spectrum of momentum ranging from about one-half the pion momentum (backward decays) to slightly greater than the pion momentum (forward decays). As a result, the initial asymmetric pion profile washes out for the case of the daughter muons.
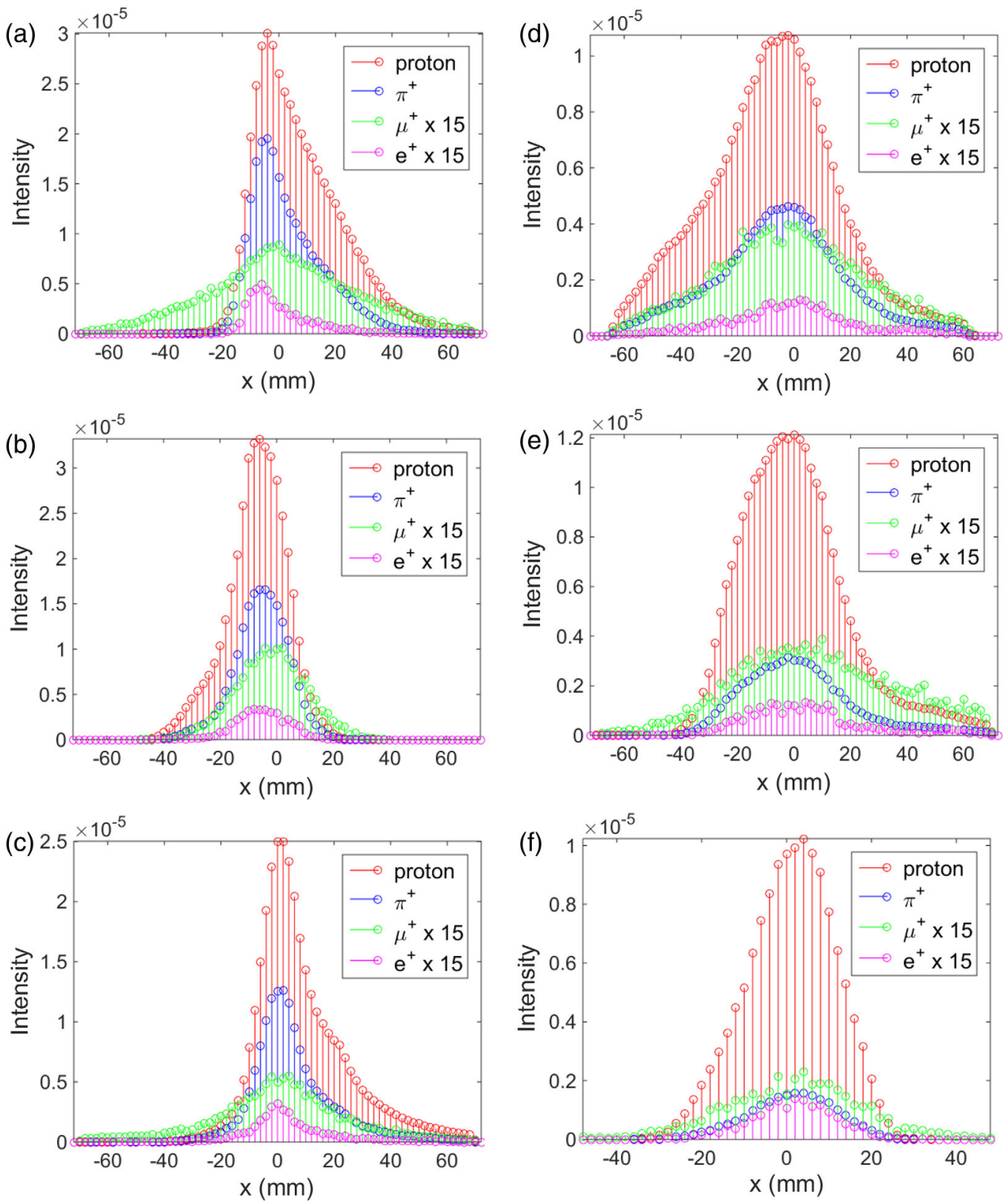

FIG. 8. Evolution of individual species of secondary particles along the M2 and M3 lines at (a) SEM804, (b) SEM810, (c) SEM706, (d) SEM711, (e) SEM726, and (f) PWC744. The vertical axis is scaled to the POT. While protons (red) dominate by at least an order of magnitude all other species, the profiles for both protons and pions (blue) resemble similar features. For visual purposes, the number of muons (green) and positrons (magenta) is multiplied by a factor of 15. 


\section{Secondary beam separation line (delivery ring)}

A complete removal of protons before injection into the storage ring of the Muon $g-2$ experiment is required in order to reduce contamination. This is accomplished with the 505-m-long DR, wherein an abort kicker magnet [24] that is strategically located in one of the DR straight sections extracts the proton beam. Both proton and muon bunches retain the length distribution of the primary beam from the recycler, where the two types of bunches almost overlap as they enter the DR. The nominal DR revolution times are 1685.5 and $1760.2 \mathrm{~ns}$ for $3.1 \mathrm{GeV} / c$ muons and protons, respectively, whereby the gap size between the two bunches increases by 75 ns per turn. Given that the abort kicker magnet rise time is $\sim 180 \mathrm{~ns}$ with a plateau of $\sim 400 \mathrm{~ns}$, the beam needs to circulate the DR at least four times before protons can be removed without any muon losses. Therefore, the default operating point when the Muon $g-2$ experiment is taking data is to circulate the DR four times. The proton-free secondary beam is then extracted into the M4 and M5 lines.

Next, we examine the reproducibility of the circulating beam in the DR. In order to accomplish this, we measure the beam profiles at a single location for multiple turns. In particular, Fig. 9 displays the measured horizontal and vertical beam profile at PWC607 for the first four turns. A notable feature of our measurements is that all profiles are overlapping each other, suggesting that the beam distribution remains unchanged from turn to turn. This is consistent with the expectations from the design, wherein the DR should be a closed (periodic) lattice. Note that a similar test was carried out further upstream of the line using PWC 301, which revealed no perceptible difference in the individual profiles as well.

As noted earlier, under normal operations for the Muon $g-2$ experiment only four turns in the DR are required.
A special run was performed in which the number of revolutions in the DR was scanned from four to 100 with the goal to measure the muon rate over a larger number of turns. The experiment was carried out as follows: First, protons were removed during the fourth turn using the abort kicker. Then, the number of revolutions was progressively increased, and, for a discrete set of turns, the beam was sent towards the Muon $g-2$ storage ring through the M4 and M5 lines. During the process, two intensity measurements were taken, one at IC025, which is located just upstream of the entrance of the storage ring, and one inside the storage ring using its electromagnetic calorimeters [25]. Note that, after a muon decays into a positron and neutrino, the positron has insufficient energy to remain on the equilibrium $(3.1 \mathrm{GeV} / c)$ orbit in the storage ring. It curls inward where its energy is detected by one of the 24 Cherenkov calorimeters and read out by large area silicon photomultipliers. The decay positrons measured in the calorimeter provide an indirect measurement of the number of muons stored in the ring [1]. It is important to emphasize that there is a significant difference between the two aforementioned measurements. While IC025 measures the intensity of all arriving species, the calorimeter measures only storable muons. As a result, the calorimeter reading is a key parameter that governs the final transmission of muons in the DR.

To go beyond and get a quantitative prediction of the muon rates over a wide range of DR turns, we plot in Fig. 10 (squares) the decay positrons, which are measured at the calorimeters, as a function of the revolution number in the DR. For simplicity and since we are interested in relative rates and not actual values, we shall assume that the calorimeter count of decay positrons equals the muon count. In reality, the measured positrons are only about $10 \%$ of the storable muons [1]. Next, to support our
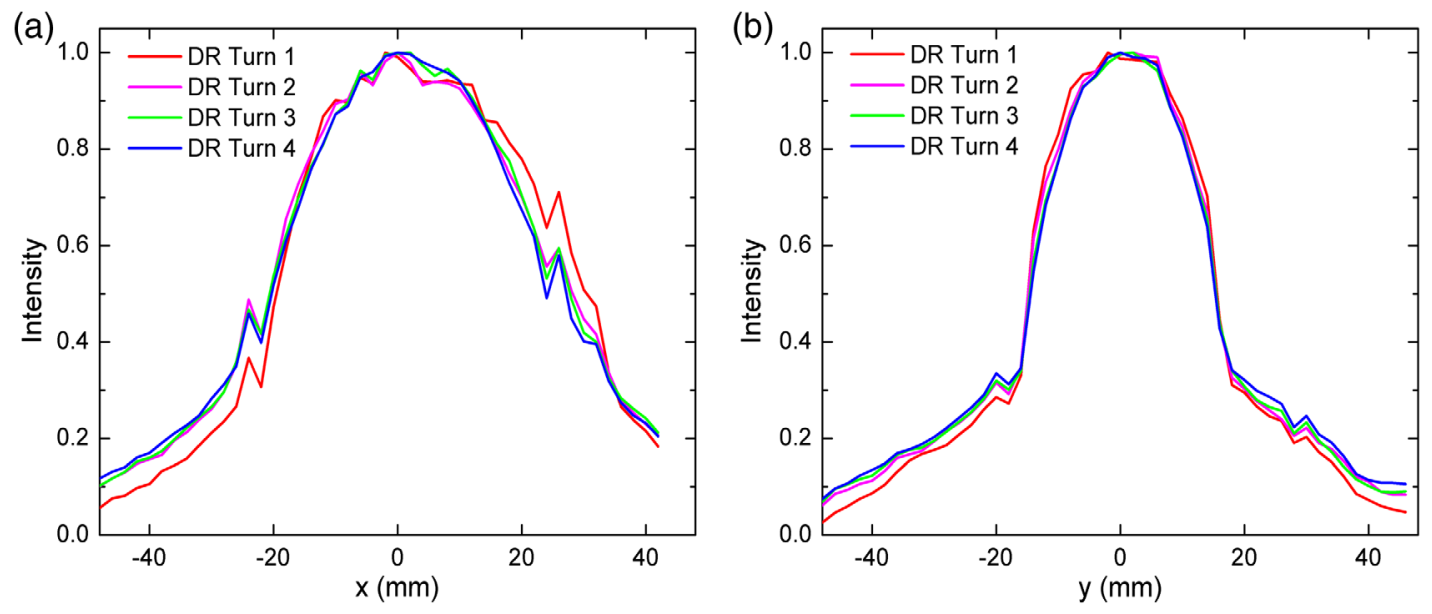

FIG. 9. Transverse beam profiles at PWC 607 for different turns along the DR of the Muon Campus. (a) Horizontal direction. (b) Vertical direction. Notice that the beam profile is reproducible from turn to turn. The kinks in the distribution are due to the discrete nature of the collected signal on the profile monitor. 


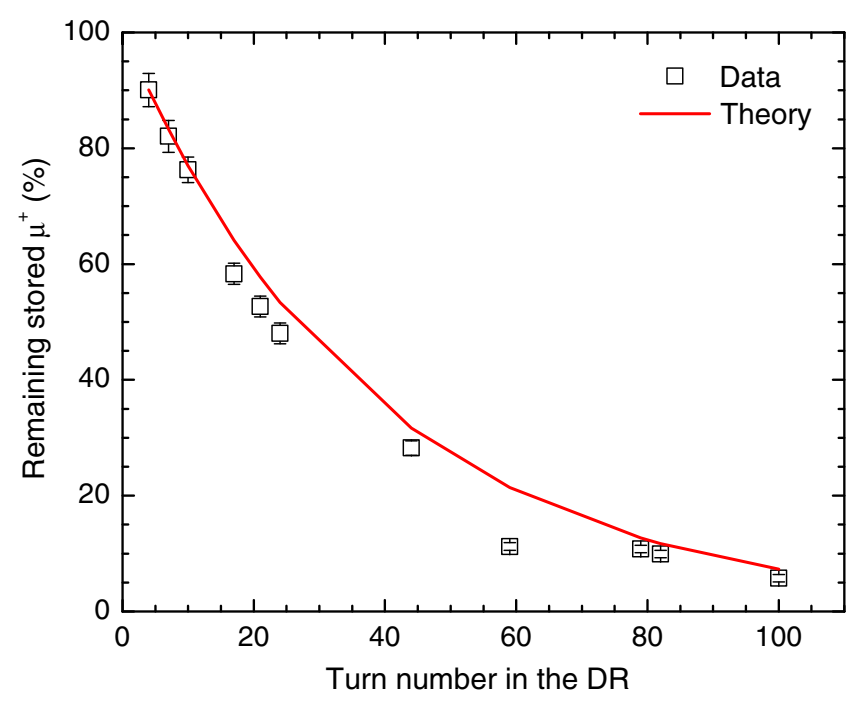

FIG. 10. Percentage of stored muons in the storage ring of the Muon $g-2$ experiment versus the number of turns in the DR. The red curve shows the number of stored muons from the exponential decay law.

measurements, we compare our results to the exponential decay expression $N=N_{0} e^{-\left(t_{\mathrm{DR}}+t_{\mathrm{M} 4 \mathrm{MS} 5}\right) / \gamma \tau_{\mu}}$, where $\tau_{\mu}=$ $2.2 \mu \mathrm{s}$ is the mean rest frame lifetime of the muon, $\gamma$ is the Lorentz factor, $N_{0}$ is the number of positrons at the calorimeters before the first DR turn begins and is 422 for our case, $t_{\mathrm{DR}}$ is the time required to circulate the DR for a given number of turns, and $t_{\mathrm{M} 4 \mathrm{M} 5}$ is the time needed to transverse the M4 and M5 lines. Note that the values in the vertical axis are the measured decay positrons scaled to $N_{0}$ and therefore can be seen as an indication of the remaining stored muons. One can see that the muon rate is strongly correlated to the number of turns in the DR, which becomes more noticeable within the first 40 turns. An interesting finding of our study is that the measured muon decay rate was not far from the exponential decay law, meaning that the error between the theory and measurement was $15 \%$ or less for all turns examined. One exemption was turn 59, wherein a timing error between the extraction from the DR and the injection to the storage ring kickers caused a significant loss of storable muons. Quantitatively, the exponential decay law predicts a muon survival rate of $8 \%$ between turns 4 and 100, while the corresponding measured value is $7 \%$.

The total intensity measured in the IC025 is illustrated in Fig. 11. While the results in Fig. 10 show a progressive muon loss on every turn, a salient feature of the data in Fig. 11 is that for some discrete number of turns the beam intensity increases. This initially surprising fact can be understood as follows: After proton extraction, the three remaining species are muons, positrons, and deuterons, and their DR revolution times are 1685.5, 1684.5, and $1969.8 \mathrm{~ns}$, respectively. While both positrons and muons nearly overlap for most of the turns, deuterons will not. This is a direct consequence of their lower relativistic

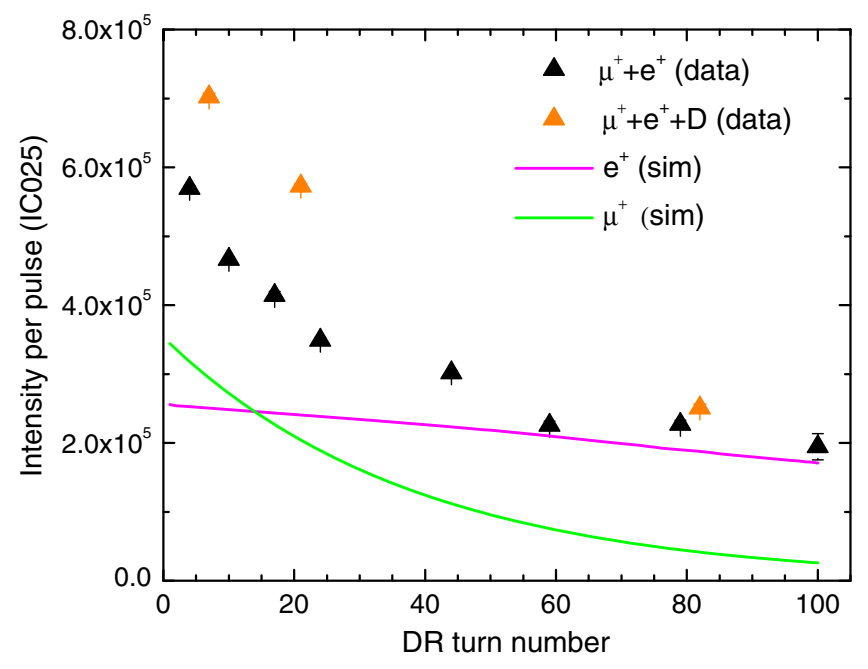

FIG. 11. Total beam intensity recorded at IC025, just upstream of the storage ring entrance of the Muon $g-2$ experiment. After the fourth turn, the beam in the DR contains a mixture of muons, positrons, and deuterons. While muons and positrons have nearly the same revolution time along the DR, deuterons have not. However, for some turns (7, 21, and 82), they will catch up with the rest of the beam, and as a result the intensity increases. The solid curves illustrate the simulated rates of muons (green) and positrons (magenta) as a function of the turn number.

factor $\gamma$, and, as a result, they will lag in time. However, for some discrete number of turns they will catch up with the rest of the beam and will be extracted to the M4 line as well. This is the reason behind the increase in intensity for turns 7,21 , and 82 .

Unfortunately, the current diagnostics in the Muon Campus allow only the measurement of the total intensity, which prohibits a priori knowledge of the transferred muon beam intensity, and this is an important quantity for evaluating the efficiency of the beam line. To circumvent this issue, we estimate the number of muons and positrons in the bunch using the data in Fig. 11. More specifically, after four revolutions, the combined measured intensity of muons and positrons is $5.69 \times 10^{5}$ per proton pulse. After 100 revolutions, the beam intensity has been reduced to $1.94 \times 10^{5}$. If we assume a $7 \%$ muon survival over 100 turns as confirmed by our aforementioned measurements, the only unknown is the transmission of positrons. With the aid of G4beamline tracking simulations, we find that $31 \%$ of muons are lost after 100 turns, primarily due synchrotron radiation. Using the above information, we estimate that, after the fourth turn in the DR, the percentage of muons at the end of the M5 beam line (IC025) is 57\% and the percentage of positrons is $43 \%$. With these numbers in mind, the following points are noteworthy: First, an independent study [26] that placed a variable-thickness $\mathrm{Pb}$ block along the beam path and then measured the positrons due to showering in the lead revealed also a ratio of 57/43 after comparing the data with simulations. 
Moreover, our G4beamline model found a ratio of $62 / 38$, which is not far from our experimental findings. The latter will be discussed in more detail in the next section.

\section{Beam transport line to the Muon $g-2$ experiment storage ring (M4 and M5 lines)}

After the removal of protons, the beam is extracted to a pair of beam lines called the M4 and M5 lines, which are illustrated in Fig. 12. The purpose of these beam lines is to transport the secondary beam from the DR to the storage of the Muon $g-2$ experiment. As shown in Fig. 12 and Table II, the beam lines contain two detectors for measuring the beam intensity: one near the beginning of the line (IC902) and one at the end (IC025). In Fig. 13, we plot the muon and positron intensities as a function of the distance along the M4 and M5 lines. The solid lines depict the ones estimated from the simulation, while the black squares depict the ones measured by the two aforementioned detectors. By carefully examining Fig. 13, the following points are noteworthy: First, from the measured values in both ICs, one can that the transmission along the M4 and M5 lines is $\sim 88 \%$, which agrees reasonably well with the model. Second, there is a near $1 / 3$ discrepancy between the measurement and simulation on the number of muons at the end of M5, with the latter predicting a higher value. While considerable beam loss in several areas is possible, the observed good transmission along the M2 and M3 lines (Fig. 6), the DR (Fig. 10), and the M4 and M5 lines (Fig. 13) makes this hypothesis less plausible. Although the precise geometry of all magnetic apertures has to be accounted for in the simulation in order to obtain a quantitative comparison, it is likely that a fraction of the beam is getting lost during injection to the DR (see Fig. 2). In that area, the ideal beam barely fits through the small apertures, and therefore any increases in the beam size, for instance, from a mismatch at the end of the M3 line as noted earlier, can cause beam loss.

To further shed light on the behavior of the M4 and M5, we measure the beam optics at various locations along the lines. To achieve this, we implement a conventional quadrupole scan technique $[27,28]$. In the physics of beams, we can often assume that the particles are bound by an ellipse expressed in terms of $\epsilon=\gamma x^{2}+2 \alpha x x^{\prime}+\beta x^{\prime 2}$, wherein the Twiss parameters $a, \beta$, and $\gamma$ describe the aspect ratio and orientation of the ellipse while $\epsilon$ describes the area of the ellipse [29]. With a quadrupole scan, a quadrupole magnet and a profile monitor are used to obtain the emittance and Twiss parameters by measuring the beam size as a function of the quadrupole magnetic field strength at some imaging station a distance $d$ further downstream [30]. If $M$ is the transfer matrix of the scanning region, created by the product of the transfer matrices of drift $S$ and quad $Q$, then the beam matrix at the screen $\sigma_{S}$ is related to the beam matrix of the quadrupole $\sigma_{Q}$ using the transformation $\sigma_{S}=M \sigma_{Q} M^{T}$, where the beam matrices in the horizontal direction are defined as

$$
\sigma_{Q}=\epsilon\left(\begin{array}{cc}
\beta_{x, Q} & -\alpha_{x, Q} \\
-\alpha_{x, Q} & \gamma_{x, Q}
\end{array}\right)
$$

and

$$
\sigma_{S}=\epsilon\left(\begin{array}{cc}
\beta_{x, S} & -\alpha_{x, S} \\
-\alpha_{x, S} & \gamma_{x, S}
\end{array}\right)
$$

Treating the quadrupole as a thin lens with focal length $f$, then

$$
Q=\left(\begin{array}{cc}
1 & 0 \\
-1 / f & 1
\end{array}\right)
$$

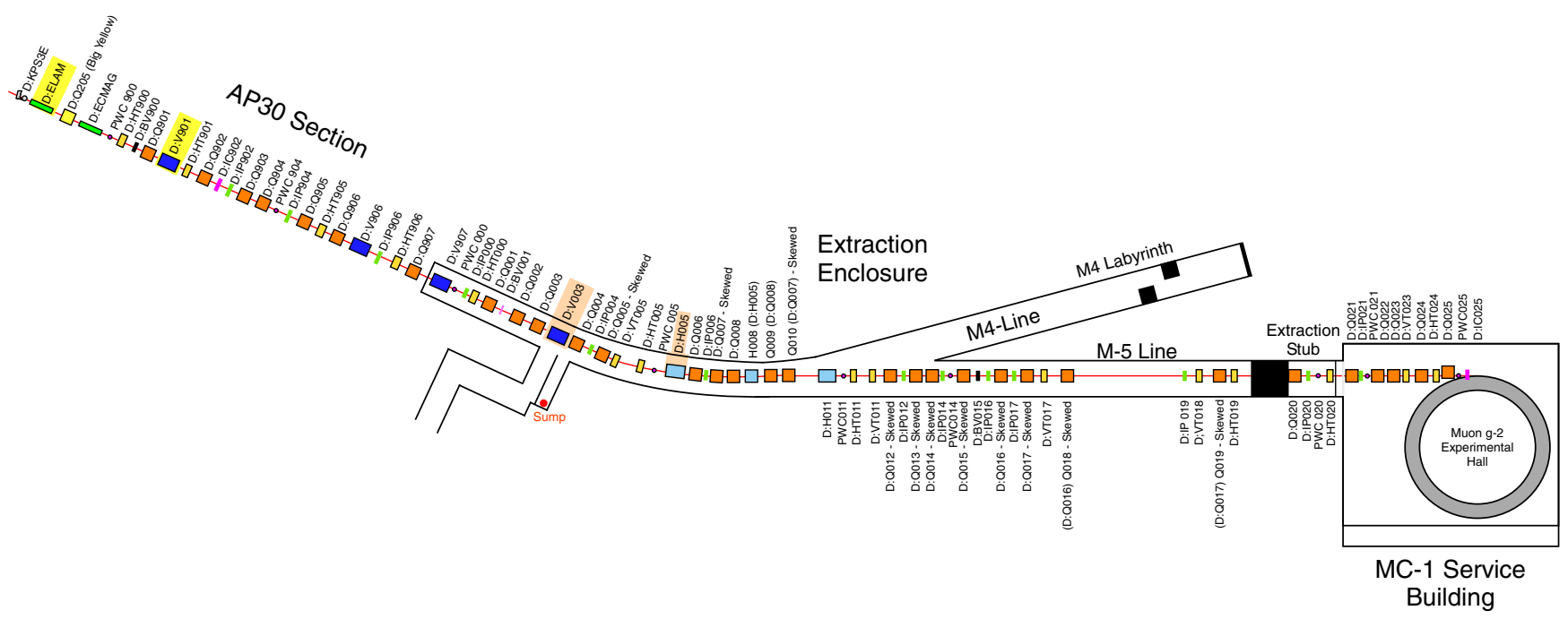

FIG. 12. Schematic layout of the M4 and M5 lines. 


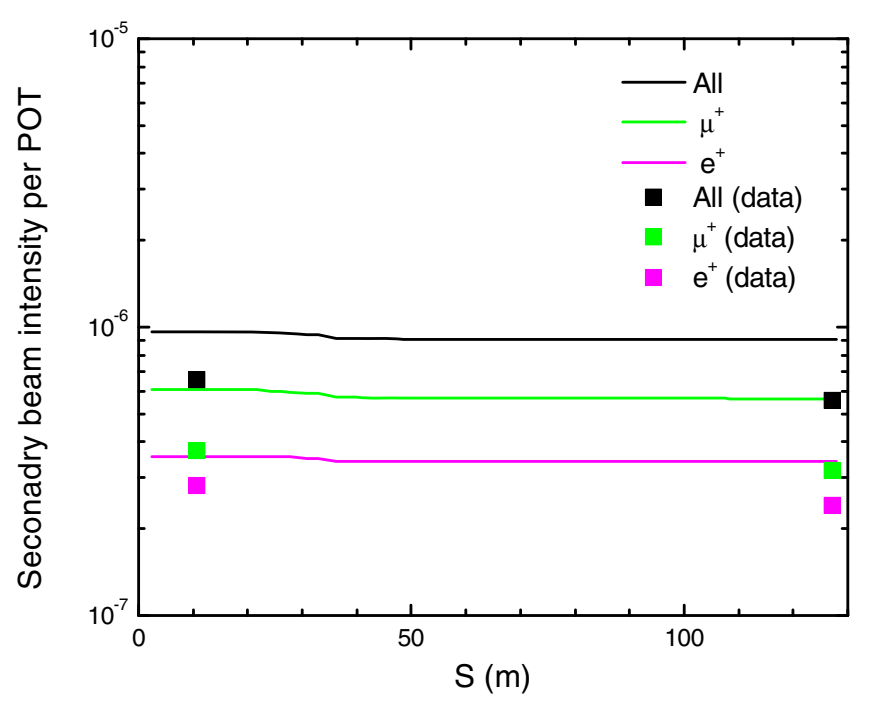

FIG. 13. Performance along the M4 and M5 lines. In black, the squares indicate the measured intensity at the entrance (IC902) and exit of the beam line (IC025), while the solid line shows the simulated total intensity for the baseline design parameters. With green and magenta, we show the corresponding rates of muons and positrons, respectively. At IC025, the simulation predicts a $\mu^{+} / e^{+}$rate of $62 / 38$.
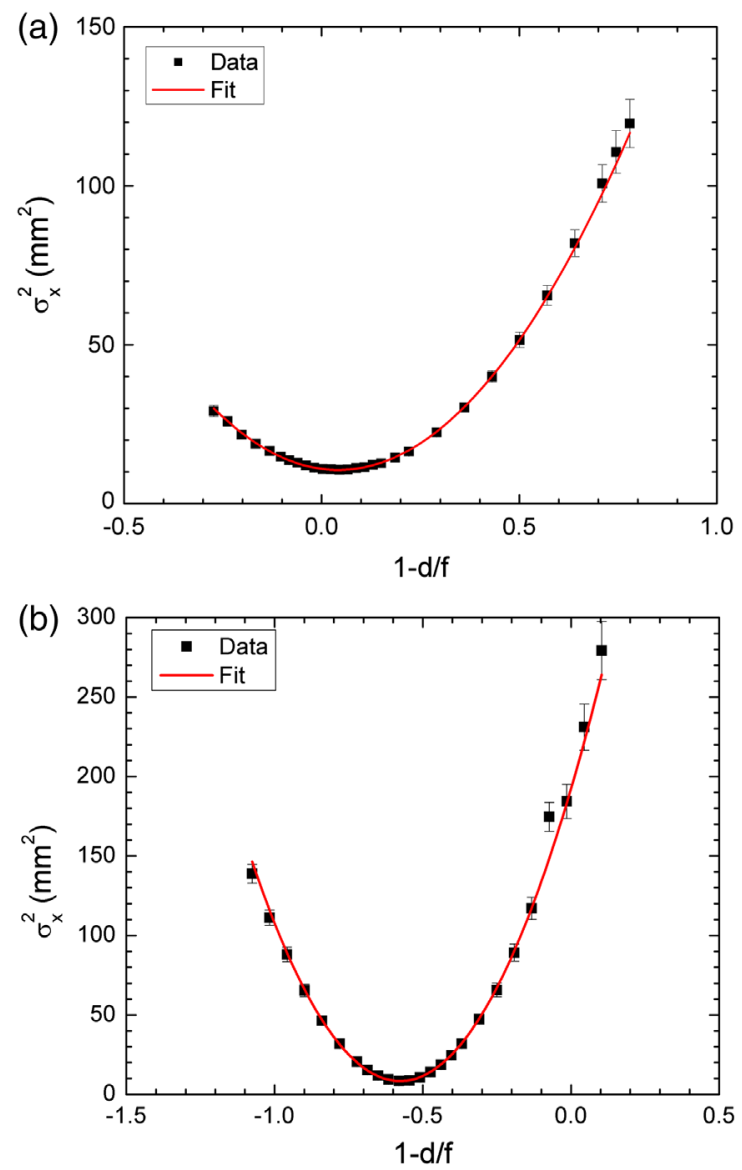

and we find the following expression:

$$
\beta_{S, x}=\beta_{Q, x}\left(1-\frac{d}{f}\right)^{2}-2 \alpha_{x, Q} d\left(1-\frac{d}{f}\right)^{2}+\gamma_{x, Q} d^{2}
$$

which can further be written in terms of the rms beam size $\sqrt{\left\langle x^{2}\right\rangle}$ at the screen:

$$
\left\langle x^{2}\right\rangle_{S}=A\left(1-\frac{d}{f}\right)^{2}-2 B d\left(1-\frac{d}{f}\right)+C d^{2},
$$

where $\quad A=\beta_{x, Q} \epsilon_{x}, \quad B=\alpha_{x, Q} \epsilon_{x}, \quad C=\gamma_{x, Q} \epsilon_{x}, \quad$ and $\epsilon_{x}=\sqrt{A C-B^{2}}$.

With this analysis in mind, the emittance and Twiss parameters can be obtained by a two-step process. First, we measure the beam size at a given drift length after a quadrupole magnet, by scanning through a range of focusing strengths. Then, we plot the rms beam size (squared) as a function of $(1-d / f)$ and apply a parabolic fitting function which will yields the three coefficients $A, B$, and $C$ and thus will fully characterize the beam Twiss parameters and beam emittance.
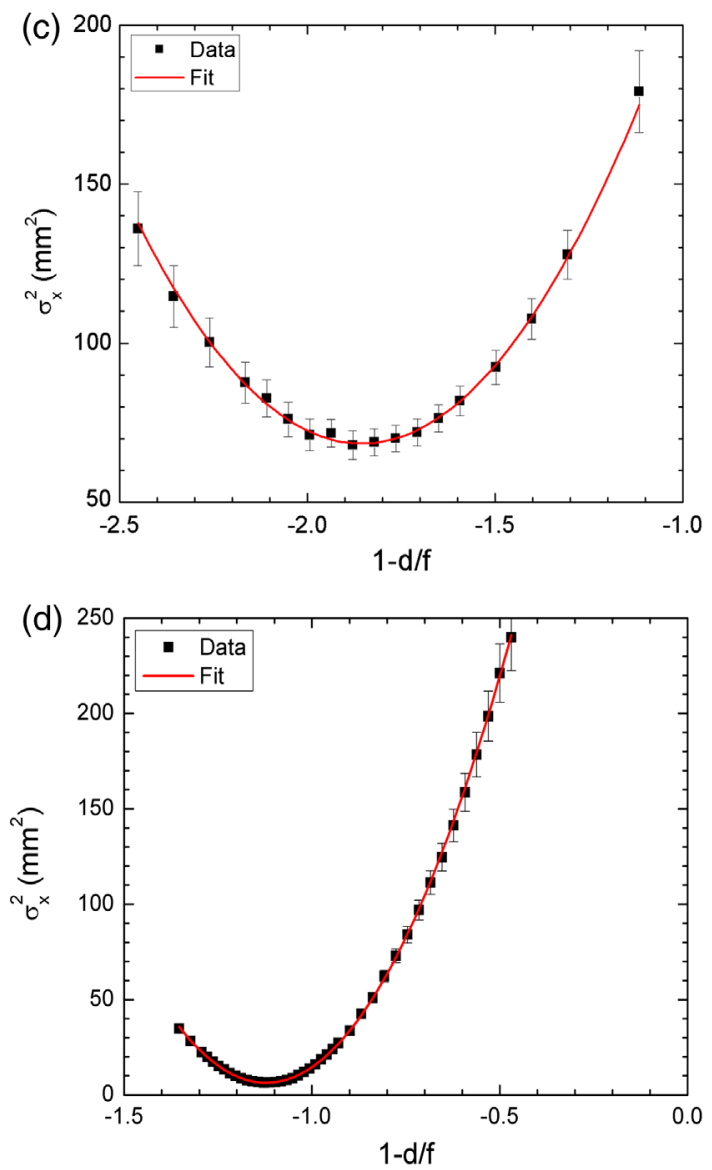

FIG. 14. Squares of rms beam size versus focusing strength using Eq. (2). (a) At PWC005. (b) At PWC014. (c) PWC021 and PWC025. The red curve is a parabolic fit to the data. 
In our case, the quadrupole scan method has been implemented at four different locations along the M5 line: just upstream of magnet Q004, just upstream of magnet Q014, just upstream of magnet Q020, and just upstream of magnet Q023. Those locations were strategically chosen to cover a broad spectrum of the M5 line. The quadrupole scan involved the collection of several transverse profiles of the secondary beam from where the rms beam size was calculated for each focal length. It is important to emphasize that, since the technique relies on fitting a parabola, special care was taken to pass through a waist during the scans in both the horizontal and vertical planes. An unfortunate implication of this was that, at some locations, a flipping of the quadrupole polarity would be necessary and was not feasible within the beam time available. As a result, it was possible to extract the beam emittance in both planes at Q021, while in all other locations data in the horizontal plane were collected only.

Figure 14 shows the squared horizontal rms beam size as a function of the magnet strength of Q004 [Fig. 14(a)], Q014 [Fig. 14(b)], Q020 [Fig. 14(c)], and Q023 [Fig. 14(d)]. For all cases, the red curve is a parabolic fit to the data. Next, using Eq. (2), we determine the Twiss parameters and emittance at the upstream edge of each quadrupole. The measured normalized rms emittance with their corresponding uncertainty is illustrated in Fig. 15. Notice the good agreement between the measured and simulated emittances. Quantitatively, the two emittances are within $10 \%$ for all cases examined, which suggests that the M5 line follows the design specifications closely. Moreover, the horizontal emittance is preserved along the M5 line, suggesting minor, if any,

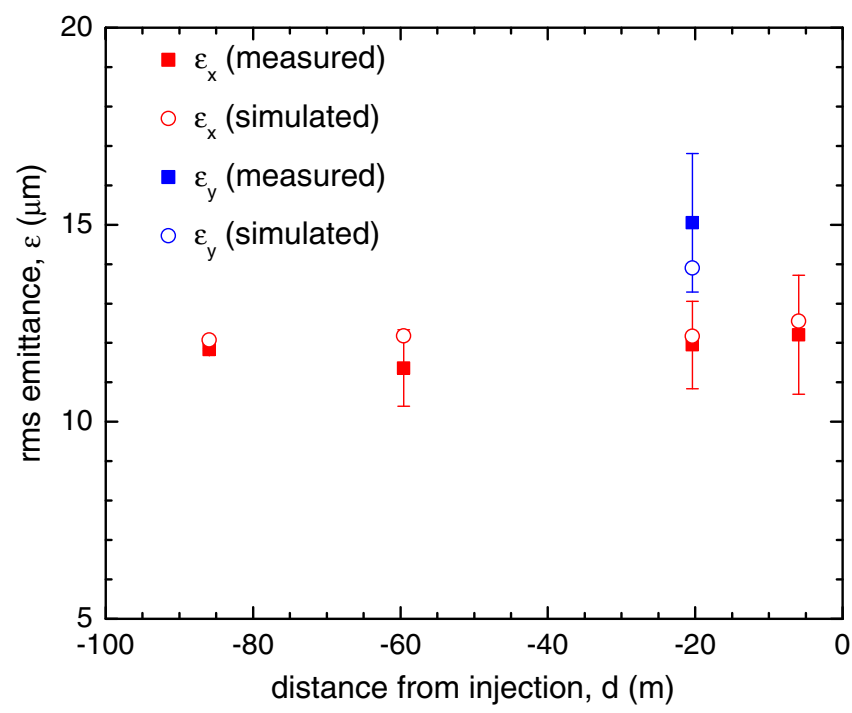

FIG. 15. Emittance evolution along the final stretch of the M5 line. Note that $d=0$ denotes the entrance of the storage ring of the Muon $g-2$ experiment. Notice that the emittance is conserved in the horizontal plane and that it agrees well with the baseline design parameters. mismatch, positioning, or field errors, which are typically associated with substantial emittance growth. This finding aligns well to the good agreement in transmission from the results in Fig. 13.

\section{SUMMARY}

The Muon Campus contains a target section wherein secondaries are produced, the delivery ring, which separates the muons from the rest of the beam, and a set of beam lines that transports them to the storage ring of the Muon $g-2$ experiment. In this paper, we have reported the first experimental results and experience gained from commissioning the Fermilab Muon Campus. We have provided more emphasis on the milestones that have demonstrated the Muon Campus capability of delivering a beam to the Muon $g-2$ experiment. These milestones included the interaction of the primary proton beam with the target, the generation and transport of muons over long sections, the monitoring of secondaries from the available diagnostics, and the development of techniques for measuring the beam transverse optics.

In this study, we have demonstrated the successful transport of the primary beam to the production target with a spot size that is adequate enough to produce a secondary beam within the baseline design specifications. For the 280-m-long pion decay line following the target, we found good agreement between measured and baseline design parameters as was indicated by a comparison between individual beam profiles and intensities along the line. In the DR, the similarity among beam profiles for different turns suggested a fairly reproducible beam as would be expected by design. The performance of the DR was further cross-checked by studying the muon rates over a wide range of turns, wherein we found that after 100 turns $7 \%$ of the muons survive while the exponential decay law predicts $8 \%$. We carried out a simple experiment to estimate the concentration of muons arriving in the storage ring, and we found the muon-to-positron rate to be 57 to 43 , which is in reasonable agreement with previous experiments [26] as well as with our simulation model. We have also established a procedure to measure the Twiss parameters along the final section of the Muon Campus, and our findings agree well with the baseline design parameters. Most importantly, we found that the beam emittance is conserved in the horizontal plane, suggesting a fairly linear beam behavior along the last stretch of the Muon Campus providing so the basis for a smooth injection to the storage ring of the Muon $g-2$ experiment. Since we found that approximately $2 / 3$ of the baseline-design beam was delivered to the storage ring, the emphasis of the beam tuning hereafter will be focused on adding more diagnostics instrumentation to further improve orbit control during transport in the M2 and M3 lines as well as on improving the modeling of the magnetic apertures, especially along the injection to the DR region. These improvements, in 
combination with our deepened understanding of lattice properties and various motions of the beam through the previous runs, are expected to improve the performance, significantly.

\section{ACKNOWLEDGMENTS}

The authors are grateful to J. Annala, M. Berz, M. Convery, J. Crnkovic, A. Ramirez, D. Still, D. Tarazona, and S. Werkema for many useful discussions. This work is supported by Fermi Research Alliance, LLC under Contract No. DE-AC02-07CH11359 with the United States Department of Energy.

[1] J. Grange et al., Muon $(g-2)$ technical design report, arXiv:1501.06858.

[2] J. Mott, The Muon $g-2$ experiment at Fermilab, Nucl. Part. Phys. Proc. 287, 65 (2017).

[3] W. Gohn, The muon $g-2$ experiment at Fermilab, arXiv:1611.04964.

[4] http://muon.fnal.gov.

[5] D. Stratakis et al., Performance analysis for the new $g-2$ experiment at Fermilab, in Proceedings of IPAC 2016, Busan (JACow, Busan, Korea, 2016), p. 996.

[6] D. Tarazona et al., Muon beam tracking and spin-orbit correlations for precision $g-2$ measurements, in Proceedings of IPAC 2016, Busan (JACow, Busan, Korea, 2016), p. 3397.

[7] M. Korostelev et al., Update on bmad simulations from target to storage ring for the new Muon $g-2$ experiment at Fermilab, in Proceedings of IPAC 2017, Copenhagen, Denmark (JACow, Copenhagen, Denmark, 2017), p. 791.

[8] G.W. Bennett et al., Final report of the E821 muon anomalous magnetic moment measurement at BNL, Phys. Rev. D 73, 072003 (2006).

[9] E. Hubbard et al., Booster synchrotron, Fermilab Technical Memo No. TM-405, 1973.

[10] B. C. Brown, P. Adamson, D. Capista, W. Chou, I. Kourbanis, D. K. Morris, K. Seiya, G. H. Wu, and M.-J. Yang, Fermilab main injector: High intensity operation and beam loss control, Phys. Rev. ST Accel. Beams 16, 071001 (2013).

[11] J. Morgan, Fermilab Technical Report No. Pbar Note 683, 2003.
[12] S. Nagaitsev, Fermilab antiproton source, recycler ring, and main injector, arXiv:1408.0759.

[13] G. Venanzoni, The New Muon $g-2$ experiment at Fermilab, Nucl. Part. Phys. Proc. 273, 584 (2016).

[14] D. Stratakis, M. E. Convery, C. Johnstone, J. Johnstone, J. P. Morgan, D. Still, J. D. Crnkovic, V. Tishchenko, W. M. Morse, and M. J. Syphers, Accelerator performance analysis of the Fermilab Muon Campus, Phys. Rev. Accel. Beams 20, 111003 (2017).

[15] M. D. Church and J. P. Marriner, The antiproton sources: Design and operation, Annu. Rev. Nucl. Part. Sci. 43, 253 (1993).

[16] B. Drendel, G Minus 2 Experiment Document No. 4590, 2016, https://gm2-docdb.fnal.gov/.

[17] https://mars.fnal.gov/.

[18] http://www.muonsinternal.com/muons3/G4beamline.

[19] http://geant4.cern.ch/.

[20] http://mad.web.cern.ch/mad/.

[21] D. Stratakis et al., Towards commissioning the Fermilab muon $g-2$ experiment, in Proceedings of IPAC 2017, Copenhagen, Denmark (JACow, Copenhagen, Denmark, 2017), p. 4505.

[22] D. L. Rubin et al., Muon beam dynamics and spin dynamics in the $g-2$ storage ring, in Proceedings of IPAC 2018, Vancouver, BC, Canada (JACow, Vancouver, BC, Canada, 2018), p. 5029.

[23] S. Romanov (private communication).

[24] J. Morgan, G Minus 2 Experiment Document No. 1957, 2014, https://gm2-docdb.fnal.gov/.

[25] A. Chapelain, The Muon g-2 experiment at Fermilab, arXiv:1701.02807.

[26] R. Fatemi, G Minus 2 Experiment Document No. 10836, 2018, https://gm2-docdb.fnal.gov/.

[27] M. Minty and F. Zimmermann, Measurement and Control of Charged Particle Beams (Springer-Verlag, Berlin, 2013).

[28] J. Bradley, B. Drendel, and D. Stratakis, First measurement of traverse beam optics for the Fermilab Muon Campus using a magnet scanning technique, Nucl. Instrum. Methods Phys. Res., Sect. A 903, 32 (2018).

[29] D. A. Edwards and M. J. Syphers, An Introduction to the Physics of High Energy Accelerators (Wiley, New York, 1993).

[30] S. G. Anderson, J. B. Rosenzweig, G. P. LeSage, and J. K. Crane, Space-charge effects in high brightness electron beam emittance measurements, Phys. Rev. ST Accel. Beams 5, 014201 (2002). 